\title{
The Marketing Of Body Image: A Cross-Cultural Comparison Of Gender Effects In The U.S. And China
}

R. Stephen Parker, Missouri State University

Diana L. Haytko, Missouri State University

Charles M. Hermans, Missouri State University

\begin{abstract}
Recently, there has been increasing controversy about the use of extremely thin female models and extremely muscular male models in advertising and fashion campaigns. While this practice has been studied extensively over time, it is again in the forefront as models with low body mass indexes are being kept off runways around the world. While most consumers understand that these images are nearly unattainable, they do tend to compare themselves with these idealized images resulting in lower satisfaction with their own bodies and lower self-esteem. While past research most often examines these feelings with respect to women, there is little research on the effect on men and their self-image. Also, while this has primarily been a "western" problem, it is becoming a problem in "eastern" cultures as western images and advertising campaigns proliferate. This study examines both U.S. and Chinese men and women's perceptions of body image. The findings show cultural differences between the U.S. and Chinese males and females. The U.S. women were the most affected, while there was no difference by gender between the Chinese men and women. However, as the Chinese culture becomes more influenced by western ideals, this trend in body image dissatisfaction should increase.
\end{abstract}

\section{INTRODUCTION}

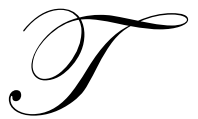

he marketing of body image is once again headline news. Recently, at a fashion show in Spain, female models not meeting a particular Body Mass Index (BMI) were not allowed to participate in runway shows (Hellmich 2006). Around the world, body mass index (BMI) measures are being taken and models with low BMIs are being banned (Mustafa 2007). Almost weekly, People magazine has a story about models, actresses or singers accused of having eating disorders because they appear dangerously thin. Many academics and practitioners feel that these individuals portray an unrealistic and dangerous image for those who compare themselves to what they see in magazines and on television. While some research suggests that women can counter-argue against these images when exposed to them in magazines (Stevens and Maclaran 2005), significant attention is now being given to how these images may be the cause of a variety of eating disorders within some groups (most notably teen girls). At the same time, little attention is being given to how these images may affect other important groups, such as men or people from developing countries just beginning to face these images on a daily basis.

Advertising which uses exceptionally thin female models and extremely muscular male models presents an image to the consumer of "perfection" that the average consumer is unlikely to achieve. Even though this standard is unattainable, Social Comparison Theory (Festinger 1954) suggests that it is still likely that individuals will compare or evaluate themselves with these idealized images that they see presented in the media. The result of this comparison is an increase in negative feelings as related to personal body image and a corresponding drop in individuals overall self esteem. Richins (1991) studied advertising that featured very attractive models and found that these models set an individuals' personal evaluation standard, leading to lower levels of personal satisfaction with their own body image among respondents. Interestingly, Richins further argues that the basis of the marketing 
concept is that marketers should attempt to provide products and services, which increase customer satisfaction levels. However, it would seem that in an effort to sway consumer's long run satisfaction and persuade them to purchase products that will enhance their appearance, marketers are deliberately using images of models whose beauty and strength is unattainable for most consumers, at the expense of the consumer's short-term level of satisfaction.

There seems to be little argument that these social comparisons may have harmful side effects in terms of a reduction in body image satisfaction and self-esteem in women. However, while much has been written about the harmful effects that idealized images have on women; little has been done to examine the consequences of marketing idealized body images to men. It is important to note that over the past three decades, males are being exposed to an increasing number of articles and advertisements which stress physical fitness and muscularity and that there is increasing sociocultural pressure to move from a thin body style to a more firm and muscular shape (Petrie et al. 1996). Given that Social Comparison Theory argues for evaluating oneself based upon images presented in the media, one would likely expect that men may well fall into the same dissatisfaction trap as have women as they are exposed to larger numbers of images and advertisements emphasizing muscular body shapes. Because women and men are exposed to different levels and content of media related to body image, we might expect some inherent attitudinal gender differences. In sum, these images should have an affect on both women and men. Yet another impact may be seen cross-culturally as advertising differs in quantity, content, and types of models to portray desirable body image concepts. Developing countries that are only now having the proliferation of western advertising images may face the same problems that accompany the use of these images that have been seen in the United States.

As the world grows smaller due to the wide spread dissemination of information through various media, it is thought that pressures previously experienced primarily in western cultures may be brought to bear on eastern cultures as well. For example, Yang, Gray, and Pope (2005) argue that past studies have shown that increased exposure to western advertising may have caused women in eastern cultures to become more dissatisfied with their body image; the same exposure may be affecting eastern males in a similar fashion. They suggest that this dissatisfaction may lead to increases in muscle dysmorphia, steroid abuse, and other body image disorders. The study reported in this paper is designed to examine whether this assumption is correct, and if so, what possible outcomes might occur. Specifically, we will examine and compare the perceptions of females and males in the United States and China to determine if significant cross-cultural differences exist in the way body image is viewed.

\section{U.S. FEMALE VIEWS AND EFFECTS}

Morrison, Kalin and Morrison (2004) addressed the underlying assumptions of Social Comparison Theory as it relates directly to women. These authors suggest that women become dissatisfied with their body image as a result of three basic assertions. These assertions are reported to be: 1) individuals have a need or drive to evaluate their opinions and abilities; 2) individuals compare their opinions and abilities to other individuals; and 3) social comparisons tend to be made with individuals one sees as being similar. It is important to note that Morrison, Kalin and Morrison (2004) report that the underlying assumptions of Social Comparison Theory have been modified in the following ways. First, the comparison point used by an individual may be someone that is very different from them. Secondly, social comparisons may include characteristics such as physical appearance or eating habits. In this case it is thought that if one is making an "upward" comparison (comparing themselves to someone in a higher status group) the result will likely be an increased dissatisfaction with their position. Conversely, if the comparison is "downward" (comparing themselves with someone in a lower status group) there should be an increase in one's positive feelings. It is also important to note that Wheeler and Miyake (1992) report that when social comparisons are made in regard to physical appearance, the direction tends to be "upward" rather than "downward," which results in lower levels of self-perception in terms of attractiveness. Turner and Hamilton (1997) also found that when women viewed models in fashion magazines (upward comparison) they reported a desire to weigh less, were less satisfied with their bodies, more frustrated with their weight, expressed more interest in being thin, and were more afraid of getting fat than those who were exposed to news magazines. More recently, Morrison, Kalin and Morrison (2004) concluded that the resulting effects of this "upward" comparison were that women tended to engage in traditional dieting practices as well as much more harmful weight control practices. 
While most of the effects of social comparison seem to be negative, the tide may be shifting amongst some consumers. The recent campaign for Dove, which used "real" models rather than the traditional model, saw a sales increase of 700\% in the first half of 2004 (Milner, 2005). It just may be that some advertisers are indeed considering the marketing concept as something to be taken seriously. Understanding how marketing efforts effect female image satisfaction is important not only to understand its effect on women, but also to understand how images of women may effect male body image satisfaction. Gulas and McKeage (2000) indicate that given the number of women that appear in advertisements aimed at men, it is possible that the male viewer's image of himself is affected by the female's images to which he is exposed. They further note that while men have been generally more satisfied with their body image, the increasing pressure seen in marketing efforts to appear physically attractive and muscular may result in a change in body image perception. Baird and Grieve (2006) also believe that the same social pressures which influence women to strive toward a thin body will likely effect men, although for men, the pressure will be toward developing a muscular body.

\section{U.S. MALE VIEWS AND EFFECTS}

As noted earlier, when social comparisons are made in an "upward" manner, the outcome is likely to be negative. Morrison, Kalin and Morrison (2004) report that when males exhibit "upward" comparison tendencies, the outcomes may include dieting to gain weight, the use of pathogenic weight control practices, and the use of steroids as a method to gain muscle mass. This observation is of particular interest in that Kolbe and Albanese (1996) found that advertisements aimed at a male audience depicted body images that are not that of the "average" male. In fact, many of the depictions were of males with very strong muscular bodies, and they were presented in an upscale conservative manner representing financial success. They further concluded that men do make social comparisons to the images they see in advertising and that these "upward" comparisons tend to affect selfperception and specifically self-esteem, more so than having a negative effect on perception of physical body image.

The frequency of "undressed" male models in advertising seems to be on the rise. Yang, Gray, and Pope (2005) reported that in the 1950s only about 3\% of male models were "undressed" however, by the 1990s that percentage had risen to approximately 35\%. As males become more exposed to advertising depicting a more muscular body image, the effects are likely to be less than positive. For example, in a recent study of 104 male college students, it was found that even brief exposures to advertising which show muscular male models led to decreases in body image satisfaction (Lorenzen, Grieve, and Thomas 2004). The authors suggest that men are becoming more dissatisfied with their bodies as a more muscular body type is becoming the "ideal" body. They further conclude that results of this increase in dissatisfaction may be lower levels of self-esteem, depression, guilt, stress, insecurity, shame, and body dissatisfaction. In turn, these negative results may lead to men attempting to gain muscle mass to attain an unrealistic muscular body image, thereby increasing their risk of developing a medical condition (eating disorder) known as muscle dysmorphia.

Hatoum and Belle (2004) also indicate that as cultural standards have shifted to value a larger and more muscular physique, men are becoming more concerned about changing their bodies to obtain this ideal image. In a study of 89 male college students, they found that $80.9 \%$ reported a desire to have a different weight than their own. They further reported a desire to have a more muscular upper body and legs. Interestingly, one-third of the sample had taken some form of dietary supplements in an attempt to build muscle mass. Higher levels of male oriented magazine readership were also found to be associated with a desire to improve ones body through an increase in building muscle mass. In a study of 173 college males, Baird and Grieve (2006) found that, when the sample was exposed to pictures of muscular men, the sample reported having lower levels of satisfaction with their bodies than those who were shown pictures of "normal" male body images. They conclude that there is support for the assertion that social comparison typically takes place in an "upward" manner which will lead to feelings of dissatisfaction which may result in a variety of medical disorders.

It is interesting to find that not all studies indicate that negative upward comparisons will take place when confronted with the idealized images that are often portrayed in the media. Elliott and Elliott (2005), using a qualitative methodology, found that most of the males that were interviewed were generally content with their appearance and while they did "admire" the muscular bodies shown in advertisements, they did not feel a reduction 
in self-esteem or an issue with self-image. The respondents felt the use of the "average" male image was less threatening and would be more effective in advertising, as it would be easier for them to relate to the "average" guy.

\section{CROSS-CULTURAL EFFECTS}

The Chinese economy, and therefore Chinese consumers, clearly represents a culture in transition. The Chinese, along with many other Eastern Asian countries, have traditionally been thought of as having collectivist tendencies, which cause the individual to conform to the whole. On the other end of the spectrum, Western countries, such as the United States, have traditionally been thought of as being individualistic in nature where people are free to express their identities in almost any way they please. However, literature suggests that as a country's economy begins to flourish and the inhabitants become more affluent, collectivist cultures tend to become more individualistic in nature (Hofstede 1980, and Triandis 2004) resulting in a somewhat "looser" society than previously existed. There seems to be little doubt that the Chinese economy fits this description and that the Chinese consumer is under significant pressure from Western advertisements to begin to express their own individualistic personalities through the products they purchase and the way they present themselves.

While it is clear that Western products are widely accepted, it is not so clear what impact Western commercials may be having on the Chinese psyche. Indeed, Gordon (2004) reports that the "Westernization" of cultures is resulting in a more global consumer, therefore countries that had previously been considered to be exempt from eating disorders (China, India, South Korea, Argentina, Brazil) are now facing the same problems as Western cultures.

When examining the evolving Chinese culture, Prendergast, Yan and West (2002) recount that Confucian beliefs suggest that ones external appearance should be much less important to overall happiness than ones ability to conform to cultural norms. They further express that many Chinese Gods and good luck charms are portrayed as being fat and that obesity, in the past, has been considered a sign of wealth and financial success. It would seem that if Chinese consumers still held these beliefs, the effects of Western advertising, which use thin models, would have very little effect and may in fact be completely ineffective. However, this may not be the case at all. Literature does exist that indicates Western advertising is having an effect on Chinese consumers.

As in the United States, most studies have examined the effects that these advertisements have had on females. However, as noted earlier, Baird and Grieve (2006) indicate it is likely that the same social pressures which influence women to strive toward a thin body will likely effect men, although the pressure will be toward developing a muscular body. For example, Lee et al. (1996), using a sample of 1,581 Chinese subjects, found that male subjects wished to be taller and possess more upper body strength while female subjects wished to weigh less and expressed dissatisfaction with the size of their stomach, waist, hips, and thighs. They further concluded that these concerns represent a more "Western" pattern of body dissatisfaction amongst Chinese consumers in Hong Kong than would have been found earlier. This conclusion seems to have been reached, at least partially, based on what appears to be a change in the traditional Chinese view of beauty. This more traditional view has been based upon an emphasis on eyebrows, eyes, ears, nose, and the mouth. Now an emphasis seems to be placed on thinness, which traditionally inferred poverty, bad luck, or illness.

Prendergast, Yan and West (2002) also confirm that exposure to advertising and other media, such as magazines and other editorial content, are contributing factors in an increase in body dissatisfaction among Chinese consumers. This conclusion was based upon a sample of 232 females from an undergraduate business program in Hong Kong. Exposure to various types of television programming and magazine articles were measured through the use of a self-response questionnaire. The study reported direct links between television and magazine exposure with body image dissatisfaction.

Several recent studies have examined the views of Chinese males towards body image. Yang, Gray, and Pope (2005) found a significant difference between the ideal body image and the actual body image of Chinese males. Specifically, they found that Chinese men chose a body image that was more muscular than they perceived themselves to possess and also indicated a significant difference between the ideal body image they thought women 
would prefer and their own perceived muscularity. Yang, Gray, and Pope (2005) concluded that although Chinese men were more comfortable with their appearance than those in the west, men in both eastern and western cultures would prefer to be more muscular.

This desire for females to be thinner and males to be more muscular appears to be taking place at a younger age. Xie et al. (2006) conducted an extensive study of Chinese adolescents. The study consisted of 6,863 students from both middle and high schools in four large Chinese cities in southern Mainland China. The study consisted of a relatively equal sample of both boys (3312) and girls (3551). The boys often described themselves as being either too thin or relatively thin while girls often described themselves as being relatively heavy or too heavy. Interestingly, children who were of normal weight and would not have been considered either under or over weight often used these descriptions. These authors concluded that exposure to either U.S. or Asian (Japan, Korea, Taiwan, and Hong Kong) advertising led to the perception of being overweight for girls and underweight for boys.

The data that is available for causes of Chinese body dissatisfaction resembles those found in literature dealing with Western cultures. It seems very reasonable to assume that the pressures being felt by the Global consumer result in more than simply feeling pressure to purchase the same products or styles of clothing. Indeed, Western advertising may well be increasingly pressuring Eastern Asian consumers to break away from their traditional perceptions and accept those being provided in the advertising messages to which they are now being exposed. Marketers must have a clearer picture of how these messages and images are being accepted if they are to effectively advertise their products in eastern cultures.

\section{THE STUDY}

It appears that there can be little doubt that social comparison does take place, both for males and females, and that it usually takes place in an "upward" manner leading to feelings of dissatisfaction or worse. It is also very clear that many advertisements are now seen on a world stage through the various media, and that pressures previously experienced primarily in western cultures are now becoming more prevalent in many developing eastern cultures. It is therefore imperative for marketers to understand how these pressures are likely to be dealt with in cultures, which have not traditionally been subject to a constant bombardment of advertising.

\section{HYPOTHESES}

H1: U.S. females will exhibit a greater perception of normative body image influences than U.S. males.

H2: Chinese females will exhibit a greater perception of normative body image influences than Chinese males.

H3: US females will exhibit a greater perception of normative body image influences than Chinese females

H4: US males will exhibit a greater perception of normative body image influences than Chinese males

\section{METHODOLOGY}

To better understand body image and self-esteem issues among females and males within the United States and China, an electronic survey was administered to a sample in each country. Since adolescents and college age groups comprise members that are most concerned with body image, we selected a sample of college students for this study. Respondents included 209 students at a large midwestern public University in the United States and 315 students at a large satellite university in Dalian, China. It is important to note that Dalian, China is located along the rapidly growing coastline of China and is also a major port for international commerce. Demographic Sample Characteristics are reported in Table 1.

The electronic survey consisted of 60 questions or statements, 11 of which dealt with body image. The scale was drawn from a study by Netemeyer, Burton and Lichtenstein (1995) and has been shown to have an alpha of .86 across items in past studies. Two of the original items from the Netemeyer, Burton and Lichtenstein (1995) scale were removed because they were written as reverse-coded items. These two items did not load with the other items in a factor analysis, which is a common problem with reverse coded items in multi-item scales (see Hersche and Engelland, 1996). The remaining 9 statements measure the extent to which a person believes that society 
pressures people to be good-looking. The scale is especially focused on women being pressured by the media to be thin. See Table 2.

Table 1: Sample Characteristics

\begin{tabular}{|c|c|c|c|c|}
\hline & & China & United States & Total \\
\hline Sample & $\mathrm{N}$ & 314 & 186 & 500 \\
& & $(100 \%)$ & $(37.1 \%)$ & $(100 \%)$ \\
\hline Gender & Male & 162 & 99 & 261 \\
& & $(32.4 \%)$ & $(19.8 \%)$ & $(52.2 \%)$ \\
& Female & 150 & 87 & 237 \\
& & $(30.0 \%)$ & $(17.4 \%)$ & $(47.4 \%)$ \\
\hline Age & $18-20$ & 13 & 20 & 33 \\
& & $2.6 \%)$ & $(4.0 \%)$ & $(6.6 \%)$ \\
& $21-23$ & 141 & 141 & 282 \\
& & $(28.2 \%)$ & $(28.2 \%)$ & $(56.4 \%)$ \\
& $24-26$ & 131 & 15 & 146 \\
& $27+$ & $(26.2 \%)$ & $(3.0 \%)$ & $(29.2 \%)$ \\
& 28 & $(1.8 \%)$ & $(7.4 \%)$ \\
\hline
\end{tabular}

Table 2: Body Image Items

Image Q1. Today's society places pressure on women to maintain a thin body.

Image Q2. Thin women receive greater social rewards than women who are not thin.

Image Q3. There is definitely a cultural expectation for women to be thin.

Image Q4. The media (advertising, tv shows, magazines) favors women with thin bodies.

Image Q5. Many people feel pressure to try to look like the people portrayed in fashion advertising and magazines.

Image Q6. Society places pressure on people to be physically attractive.

Image Q7. Good-looking people receive greater social rewards than people who are not good looking.

Image Q8. There is definitely a cultural expectation for people to be physically attractive.

Image Q9. The media (advertising, tv shows, magazines) favors people who are good looking.

Each of the items were measured on a seven-point Likert-type scale which ranged from "strongly disagree" $=1$ to "strongly agree" $=7$. The 9 items loaded together and produced a univariate factor solution for body image. A factor analysis was performed for each sub-sample and for the entire dataset separately. See Table 3 for the factor solutions and scale reliabilities.

Table 3: Factor Solution for Body Image

\begin{tabular}{|c|c|c|c|}
\hline Item & China & USA & Entire Sample \\
\hline Variance Explained & $49.00 \%$ & $64.74 \%$ & $63.52 \%$ \\
\hline Eigen value & 4.410 & 5.826 & .717 \\
\hline Cronbach's alpha & .870 & .929 & .928 \\
\hline Loadings: & \multicolumn{2}{|l|}{} \\
\hline Q1 & .647 & .731 & .766 \\
\hline Q2 & .638 & .649 & .698 \\
\hline Q4 & .632 & .787 & .768 \\
\hline Q5 & .658 & .770 & .765 \\
\hline Q6 & .672 & .791 & .809 \\
\hline Q8 & .676 & .838 & .751 \\
\hline Q9 & .674 & .701 & .792 \\
\hline
\end{tabular}

Maximum likelihood extraction with oblique rotation. 


\section{RESULTS}

The results of this study show significant differences in how respondents from the two cultures view body image. While no main effect exists for gender when controlling for country $(F=1.447, p<.065)$, there is a significant main effect for country $(\mathrm{F}=8.796, \mathrm{p}<.001)$. In addition there is a significant effect for each of the nine questions across country. The data in Table 4 show the differences in mean and p-values for each gender group for the respondents from the United States and China, on each of the nine questions. Table 5 provides additional post hoc tests to identify differences between each of the paired groups.

Table 4: Perception of Body Image

\begin{tabular}{|l|c|c|c|c|c|c|}
\hline Item & $\begin{array}{c}\text { United States Males } \\
\text { Mean (s.d.) }\end{array}$ & $\begin{array}{c}\text { United States } \\
\text { Females Mean (s.d.) }\end{array}$ & $\begin{array}{c}\text { Chinese Males } \\
\text { Mean (s.d.) }\end{array}$ & $\begin{array}{c}\text { Chinese Females } \\
\text { Mean (s.d.) }\end{array}$ & F & p-value \\
\hline Q1 & $5.38(1.34)$ & $6.07(1.18)$ & $4.02(1.39)$ & $4.20(1.47)$ & 57.095 & .001 \\
\hline Q2 & $5.40(1.27)$ & $5.48(1.34)$ & $4.33(1.48)$ & $4.25(1.38)$ & 26.354 & .001 \\
\hline Q3 & $5.38(1.28)$ & $5.94(1.13)$ & $4.29(1.48)$ & $4.38(1.30)$ & 40.631 & .001 \\
\hline Q4 & $5.86(1.18)$ & $6.24(0.91)$ & $4.65(1.50)$ & $4.62(1.35)$ & 45.826 & .001 \\
\hline Q5 & $5.67(1.15)$ & $6.07(1.07)$ & $4.41(1.40)$ & $4.57(1.30)$ & 47.198 & .001 \\
\hline Q6 & $5.80(1.13)$ & $6.27(0.81)$ & $4.36(1.38)$ & $4.47(1.30)$ & 69.653 & .001 \\
\hline Q7 & $5.60(1.20)$ & $5.74(1.13)$ & $4.34(1.48)$ & $4.34(1.43)$ & 37.069 & .001 \\
\hline Q8 & $5.64(1.09)$ & $6.07(0.93)$ & $4.37(1.41)$ & $4.26(1.26)$ & 61.497 & .001 \\
\hline Q9 & $5.96(1.17)$ & $6.35(0.76)$ & $4.45(1.49)$ & $4.60(1.28)$ & 65.996 & .001 \\
\hline
\end{tabular}

Means based on 7 point scale: $1=$ Strongly Disagree $-7=$ Strongly Agree

\section{DISCUSSION}

The data presented above indicates that there is partial acceptance for H1 (U.S. females will exhibit a greater perception of normative body image influences than U.S. males) as responses were found to be significantly different for three of the nine questions. However, no significance was found for the remaining six questions. It is however important to note that while significant differences were not found, the mean scores for each of the nine questions were all in the five to six range which indicates rather strong agreement for each of the nine questions showing agreement with the concept that society places a great deal of pressure on women to either remain or become thin, and that those women who do fit this image will be socially rewarded for doing so. There was no support found for $\mathrm{H} 2$ (Chinese females will exhibit a greater perception of normative body image influences than Chinese males) and $\mathrm{H} 2$ was therefore rejected. Chinese respondents rated each of the questions in the more neutral range, which is represented by scores, which were slightly above 4 .

Support was found for $\mathrm{H} 3$ (US females will exhibit a greater perception of normative body image influences than Chinese females) and H3 was accepted. Mean scores for U.S. females were found to fall into the high five to low 6 ranges while mean scores for Chinese females fell into the mid-four ranges. Significant differences were found for all of the nine questions indicating that U.S. females feel more pressure to be thin than do Chinese females.

Support was also found for H4 (US males will exhibit a greater perception of normative body image influences than Chinese males) and H4 was therefore accepted. Mean scores for U.S. males were in the high 5 range while mean scores for Chinese males were found to be in the low to mid-4 range. Significant differences were found for all nine of the questions indicating U.S. males felt more pressure to maintain a particular body image than did Chinese males. 
Table 5: Multiple Comparisons (Tukey's HSD)

\begin{tabular}{|c|c|c|c|c|c|}
\hline \multicolumn{3}{|c|}{ Variables } & \multicolumn{3}{|c|}{ Estimates } \\
\hline Dependent Variable & $\begin{array}{c}\text { Group } \\
\text { (I) }\end{array}$ & $\begin{array}{c}\text { Group } \\
(\mathbf{J})\end{array}$ & $\begin{array}{c}\text { Mean Diff. } \\
(\mathbf{I}-\mathrm{J})\end{array}$ & Std. Error & P-Value \\
\hline \multirow[t]{6}{*}{ Q1 } & US Females & US Males & .691 & .201 & .004 \\
\hline & US Females & Chinese Females & 1.870 & .185 & .001 \\
\hline & US Females & Chinese Males & 2.050 & .182 & .001 \\
\hline & US Males & Chinese Females & 1.179 & .179 & .001 \\
\hline & US Males & Chinese Males & 1.359 & .175 & .001 \\
\hline & Chinese Females & Chinese Males & .180 & .156 & .658 \\
\hline \multirow[t]{6}{*}{ Q2 } & US Females & US Males & .085 & .204 & .976 \\
\hline & US Females & Chinese Females & 1.229 & .188 & .001 \\
\hline & US Females & Chinese Males & 1.154 & .185 & .001 \\
\hline & US Males & Chinese Females & 1.145 & .181 & .001 \\
\hline & US Males & Chinese Males & 1.069 & .178 & .001 \\
\hline & Chinese Females & Chinese Males & -.076 & .158 & .964 \\
\hline \multirow[t]{6}{*}{ Q3 } & US Females & US Males & .566 & .195 & .021 \\
\hline & US Females & Chinese Females & 1.566 & .179 & .001 \\
\hline & US Females & Chinese Males & 1.656 & .176 & .001 \\
\hline & US Males & Chinese Females & 1.001 & .174 & .001 \\
\hline & US Males & Chinese Males & 1.090 & .171 & .001 \\
\hline & Chinese Females & Chinese Males & .089 & .152 & .936 \\
\hline \multirow[t]{6}{*}{ Q4 } & US Females & US Males & .381 & .191 & .192 \\
\hline & US Females & Chinese Females & 1.615 & .176 & .001 \\
\hline & US Females & Chinese Males & 1.586 & .173 & .001 \\
\hline & US Males & Chinese Females & 1.234 & .170 & .001 \\
\hline & US Males & Chinese Males & 1.205 & .167 & .001 \\
\hline & Chinese Females & Chinese Males & -.029 & .149 & .997 \\
\hline \multirow[t]{6}{*}{ Q5 } & US Females & US Males & .398 & .187 & .144 \\
\hline & US Females & Chinese Females & 1.497 & .171 & .001 \\
\hline & US Females & Chinese Males & 1.662 & .168 & .001 \\
\hline & US Males & Chinese Females & 1.099 & .166 & .001 \\
\hline & US Males & Chinese Males & 1.264 & .163 & .001 \\
\hline & Chinese Females & Chinese Males & .165 & .145 & .664 \\
\hline \multirow[t]{6}{*}{ Q6 } & US Females & US Males & .475 & .179 & .041 \\
\hline & US Females & Chinese Females & 1.807 & .165 & .001 \\
\hline & US Females & Chinese Males & 1.908 & .162 & .001 \\
\hline & US Males & Chinese Females & 1.332 & .159 & .001 \\
\hline & US Males & Chinese Males & 1.433 & .156 & .001 \\
\hline & Chinese Females & Chinese Males & .101 & .140 & .888 \\
\hline \multirow[t]{6}{*}{ Q7 } & US Females & US Males & .143 & .198 & .889 \\
\hline & US Females & Chinese Females & 1.396 & .183 & .001 \\
\hline & US Females & Chinese Males & 1.399 & .180 & .001 \\
\hline & US Males & Chinese Females & 1.253 & .176 & .001 \\
\hline & US Males & Chinese Males & 1.256 & .173 & .001 \\
\hline & Chinese Females & Chinese Males & .003 & .155 & 1.00 \\
\hline \multirow[t]{6}{*}{ Q8 } & US Females & US Males & .432 & .180 & .079 \\
\hline & US Females & Chinese Females & 1.813 & .166 & .001 \\
\hline & US Females & Chinese Males & 1.701 & .164 & .001 \\
\hline & US Males & Chinese Females & 1.381 & .160 & .001 \\
\hline & US Males & Chinese Males & 1.269 & .158 & .001 \\
\hline & Chinese Females & Chinese Males & -.112 & .141 & .858 \\
\hline \multirow[t]{6}{*}{ Q9 } & US Females & US Males & .393 & .184 & .145 \\
\hline & US Females & Chinese Females & 1.750 & .170 & .001 \\
\hline & US Females & Chinese Males & 1.899 & .167 & .001 \\
\hline & US Males & Chinese Females & 1.357 & .164 & .001 \\
\hline & US Males & Chinese Males & 1.506 & .161 & .001 \\
\hline & Chinese Females & Chinese Males & .149 & .144 & .727 \\
\hline
\end{tabular}


These responses indicate that, at least to date, respondents in the United States feel that there is more pressure, than do Chinese respondents, for women to maintain a thin body image. This may of course be the result of U.S. consumers growing up in a mass media world, which has inundated them with body image advertising their entire life. It may also be that the Chinese body image has traditionally been thinner and therefore there is less pressure to reach or maintain a body type that is simply more common in the Chinese culture. It is interesting to note however, that all groups indicate that the media favors women with thin bodies and that the media favors people who are more attractive. These findings are of importance as they may well impact the way each group views their own bodies. As previously noted, Gulas and McKeage (2000) indicate that males' perceptions of how women are portrayed in the western media may well influence their perceptions of their own body images. It is logical to conclude that if males feel that females who are thin tend to be rewarded by society, males that posses a more muscular physique may also be held in higher regard than those who are less muscular.

\section{CONCLUSION}

The literature presented here indicates that Western advertising is likely to present the consumer with an unrealistic image of "perfection" that will tend to serve as a standard for what should be considered physically attractive. This image will likely have some effect on how consumers, in both Western and Eastern cultures, view themselves in comparison to those models shown in advertisements. The literature also indicates that it is likely that these advertisements will have a very real effect on the consumers' psyche as they will generally result in an "upward" comparison which is likely to result in consumer dissatisfaction.

While much of the recent press, as well as academic literature, examine the influence of advertising on women, it is important to remember that these advertisements may have similar effects on men. Kolbe and Albanese (1996) reported that males also make "upward" comparisons and the result is often a negative influence on self-esteem and self-perception. For example, it is thought that males will often exhibit this dissatisfaction through the desire to gain muscle mass, occasionally through the use of steroids, to become more physically "in shape."

Several questions arise that must be addressed by marketers in relation to the body image question. First, is it in the marketer's best interest to cause such dissatisfaction through the use of thin models in the case of women and muscular models in the case of men's advertisements? It would seem that if viewers look at a model and see that person as having a body shape that they are unlikely to achieve the viewer of the advertising would be less likely to purchase the product in question. On-the-other-hand, if viewers of advertising see clothing that looks good on models with a more reasonable body shape it would seem more likely that they would assume that the product would look good on them as well. Second, the very real question also exists that if the market does not govern itself in this matter, regulatory agencies may step in and impose regulations that the industry will have to follow. This is not an unlikely scenario to imagine. Models are presently being banned from runways unless they meet a certain body mass index, the industry is being "asked" to watch for models who are becoming too thin, and stores are now showing only clothing that is not smaller than a size eight.

A third and less pragmatic question revolves around whether or not it is ethical for western advertisers to use techniques that have proven somewhat effective, although hurtful in many ways, in developing countries. Yang, Gray, and Pope (2005) and Xie et al. (2006) have shown that western images have affected Chinese consumers of all ages in negative ways and Gordon (2004) believes that this effect has been seen in the form of eating disorders appearing in countries, which previously experienced very little problem in this area. Future research might be centered on whether or not this type of body image manipulation actually results in higher levels of sales or simply higher levels of dissatisfaction.

With respect to global marketing campaigns, advocates would say that the world is becoming so small that differences across cultures are minor. Yet, a recent study by McCarty et al. (2007) found that country differences do exist and that there are segments of consumers within a country who respond differently based on consumer style. This is important for marketers in their choice of models for advertising, while the ultra thin model may work well in the U.S., it may not work as well in China or other rapidly developing countries. Future research should examine cultural differences with respect to attitudes toward weight and it's tie to wealth. 


\section{REFERENCES}

1. Baird, Amy L. and Frederick G. Grieve (2006) Exposure to Male Models in Advertisements Lead to a Decrease in Men's Body Satisfaction, North American Journal of Psychology, 8 (1), March-April: 115-121.

2. Beardon, William O. and Randall L. Rose (1990) Attention to Social Comparison Information: An Individual Difference Factor Affecting Consumer Conformity, Journal of Consumer Research, 16 (March): 461-71.

3. Elliott, Richard and Christine Elliott (2005) Idealized Images of the Male Body in Advertising: A Readerresponse Exploration, Journal of Marketing Communications, 11 (1), March: 3-19.

4. $\quad$ Festinger, Leon (1954) A Theory of Social Comparison Processes, Human Relations, 7 (May): 117-140.

5. Gordon, Richard A. (2004) Commentary: Towards A Clinical Ethnography, Culture, Medicine \& Psychiatry, December, 28 (4): 603-606.

6. Gulas, Charles S. and Kim McKeage (2000) Extending Social Comparison: An Examination of the Unintended Consequences of Idealized Advertising Imagery, Journal of Advertising, Summer, 29 (2): 17 28.

7. Hatoum, Ida Jodette and Deborah Belle (2004) Mags and Abs: Media Consumption and Bodily Concerns in Men, Sex Roles, October, 51 (7/8): 397-407.

8. Hellmich, Nanci (2006) Do thin models warp girls' body image? USA Today, September 26, 1A.

9. Hersche, Joel and Brian Engelland (1996) Reverse-polarity items and scale unidimensionality, Journal of the Academy of Marketing Science 24 (4): 366-375.

10. Hofstede, Geert (1980) Culture's Consequences: International Differences In Work-Related Values. Beverly Hills, CA: Sage.

11. Kolbe, Richard H. and Paul J. Albanese (1996) Man to Man: A Content Analysis of Sole-Male Images in Male-Audience Magazines, Journal of Advertising, 25 (4): 1-20.

12. Lee, Sing, Tony Leung, Antoinette M. Lee, Hong Yu, and C.M. Leung (1996) Body Dissatisfaction Among Chinese Undergraduates and Its Implications for Eating Disorders in Hong Kong, International Journal of Eating Disorders, 20 (1): 77-84.

13. Lorenzen, Lisa A., Frederick G. Grieve, and Adrian Thomas (2004) Exposure to Muscular Male Models Decreases Men's Body Satisfaction, Sex Roles, 51 (11/12), December: 743-748.

14. Martin, Mary C. and James W. Gentry (1997) Stuck in the Model Trap: The Effects of Beautiful Models in Ads on Female Pre-Adolescents and Adolescents, Journal of Advertising, 26 (Summer): 19-33.

15. McCarty, John A., Martin I. Horn, Mary Kate Szenasy and Jocelyn Feintuch (2007) An exploratory study of consumer style: Country differences and international segments, Journal of Consumer Behaviour, 6 (1): 48-59.

16. Milner, Colin (2005) Older Women Want “Real” Advertising, Fitness Business Pro, 21 (10), October: 26.

17. Morrison, Todd, G., Rudolf Kalin, and Melanie A. Morrison (2004) Body-Image Evaluation and BodyImage investment Among Adolescents: A Test of Sociocultural and Social Comparison Theories, Adolescence, 39 (155), Fall: 571-592.

18. Mustafa, Nadia (2007) Now you see them, now you don't, Time, 169 (3), January 15.

19. Netemeyer, Richard G., Scott Burton and Donald R. Lichtenstein (1995) Trait Aspects of Vanity: Measurement and Relevance to Consumer Behavior, Journal of Consumer Research, 21 (March): 612-26.

20. Petrie, Trent A., Laura J. Austin, Barbara J. Crowley, Annette Helmcamp, Courtney E. Johnson, Regan Lester, Rebecca Rogers, Jeff Turner and Kevin Walbrick (1996) Sociocultural explanations of attractiveness for males, Sex Roles, 35 (9/10): 581-602.

21. Prendergast, Gerard, Leung Kwok Yan, and Douglas C. West (2002) Role Portrayal in Advertising and Editorial Content, and Eating Disorders: An Asian Perspective, International Journal of Advertising, 21 (2): 237-258.

22. Richins, Marsha L. (1991) Social Comparison and the Idealized Images of Advertising, Journal of Consumer Research, 18 (1), June: 71-83.

23. Stevens, Lorna and Pauline Maclaran (2005) Exploring the 'shopping imaginary': The dreamworld of women's magazines, Journal of Consumer Behaviour, 4 (4): 282-292.

24. Triandis, Harry C. (2004) The Many Dimensions of Culture, Academy of Management Executive, 18 (1): 88-93. 
25. Turner, Sherry L. and Heather Hamilton (1997) The Influence of Fashion Magazines on the Body Image Satisfaction of College Women: An Exploratory Analysis, Adolescence, Fall, 32 (127): 603-614.

26. Wheeler, Ladd and Kunitate Miyake (1992) Social Comparison in Everyday Life, Journal of Personality and Social Psychology, 62 (5): 760-773.

27. Xie, Bin, Chih-Ping, Chou, Donna Spruijt-Metz, Kin Reynolds, Florence Clark, Paula Palmer, Peggy Gallaher, Ping Sun, Qian Guo, and Anderson C. Johnson (2006) Weight Perception and Weight-Related Sociocultural and Behavioral Factors in Chinese Adolescents, Preventive Medicine, 42 (3): 229-234.

28. Yang, Chi-Fu Jeffrey, Peter Gray, and Harrison G. Pope, Jr., (2005) Male Body Image in Taiwan Versus the West: Yanggang Zhiqi Meets the Adonis Complex, American Journal of Psychiatry, 162 (2): 263-269. 
NOTES 